\title{
A Warm Welcome to Quasicrystals
}

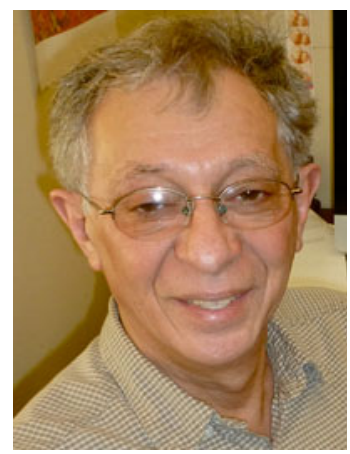

L.A. Bendersky

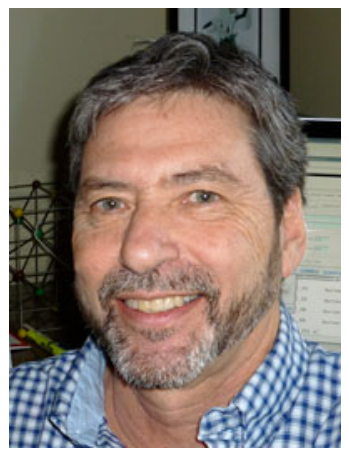

B. Burton
The ultimate recognition of quasicrystals (QC) as legitimate members of the Materials Kingdom finally arrived with the 2011 Nobel Prize in Chemistry awarded to material scientist Dan Shechtman for the discovery of an icosahedral Al-Mn phase. ${ }^{[1]}$ Before this discovery thermodynamic and structural properties of materials were studied for either isotropic substances (gases, liquids or glasses as frozen liquids; the disordered systems with infinite space group symmetry) or periodic structures (ordered, 230 crystallographic space groups). The discovery of QC significantly increased the classes of materials that must be investigated for their structural, thermodynamic and physical properties; and also to determine their (meta)stability fields in phase diagrams.

Mathematical descriptions of QCs as 3D structures with quasiperiodic lattices that can be generated by projection from higher-dimension periodic lattices (e.g. a 6D hypercube for an icosahedral phase) opened the possibility of filling the gap between isotropic and periodic structures with an infinite number of ordered non-periodic structures. "The aperiodic zoo" of tiles includes not only the famous pentagonal Penrose tile, but also tiles with higher than six-fold rotations, e.g. octagonal and seven-fold symmetries; even ordered tiling of infinite order rotations, the pinwheel tiling, is possible. ${ }^{[2]}$

The first QC was discovered as a metastable phase of icosahedral symmetry in the Al-Mn system. Since then, numerous stable and metastable icosahedral QCs have been found, mostly as intermetallic phases, but also in minerals, liquid crystals, polymers and nanoparticles. The discovery of icosahedral QCs was followed by discoveries of axial QCs (periodic in one dimension) with decagonal, pentagonal, octagonal, dodecagonal and enneagonal symmetries. ${ }^{[3]}$ The discovery of an Al-Fe-Si glass, which solidifies from melt, opens the possibility of an ordered structure of infinite rotations. ${ }^{[4]}$

Most thermodynamically stable QCs, are ternary or higher order, but some binary QCs appear to be stable as well, at least at finite temperatures. Stable QCs allow quantitative structural studies, and determinations of their thermodynamic functions and phase equilibria. The first stable icosahedral QC was synthesized in the Al-Cu-Fe ternary; a stable axial decagonal QC was discovered in $\mathrm{Al}-\mathrm{Co}-\mathrm{Cu}$; and the first stable binary icosahedral QCs were found in $\mathrm{Ca}-\mathrm{Cd}$ and $\mathrm{Yb}-\mathrm{Cd}$. The stabilities of QCs are dominated by the electron/atom ratio rather than bulk composition; thus most QCs can be considered to be Hume-Rothery compounds.

Translational aperiodicity presents fundamental challenges to electronic structure calculations (e.g. DFT) for QCs. ${ }^{[5]}$ Even loworder periodic approximants [e.g. the Frank-Kasper phase: $(\mathrm{Al}, \mathrm{Zn})_{49} \mathrm{Mg}_{32}$ ] are very complex crystal structures, typically with hundreds atoms per unit cell, and the atoms/cell ratio increases by a factor of $\approx 4.17$ for each step in the approximants hierarchy. Thus, very large complex models are typically required to obtain good approximations for QC properties; e.g. energies of formation. However, available experimental data allow measurements of QCs energetics via the same techniques that one applies to complex intermetallics.

CALPHAD assessments that include QC-descriptions have only been performed for a few systems. ${ }^{[6,7]}$ QC are typically solution phases with significant homogeneity ranges, and multiple, distinct atomic sites; so they will be poorly modeled as line compounds. Their free energies will inevitably be described with the sublattice model, ${ }^{[6]}$ and the primary constraints on fitting parameters will be experimental phase equilibria- or thermochemical-data. Currently, it is routine to calculate DFT-formation energies for most intermetallics, and for many solid solutions one can use the cluster expansion technique to approximate more detailed thermodynamic information (e.g. free energies as functions of temperature and bulk composition that can be used to fit sublattice model polynomials). For QCs, the computational task is difficult. Non-heroic DFT calculations of formation energies (or other thermodynamic functions) will only be practical only for low-order approximants. For higher-order approximants, the best option may be empirical potentials, ${ }^{[8]}$ which are significantly less accurate than DFT, but may provide useful starting values for CALPHAD optimizations.

\section{L.A. Bendersky and B. Burton}

\section{References}

1. Scientific Background on the Nobel Prize in Chemistry 2011, The Discovery of Quasicrystals, http://www.nobelprize.org/ nobel_prizes/chemistry/laureates/2011/advanced-chemistryprize2011.pdf. Last accessed 2 Feb 2012

2. M. Senechal, Quasicrystals and Geometry, Cambridge University Press, Cambridge, 1995 
3. W. Steurer, Twenty Years of Structure Research on Quasicrystals. Part I. Pentagonal, Octagonal Decagonal and Dodecagonal Quasicrystals, Z. Kristallogr., 2004, 219, p 391-446

4. J.W. Cahn and L.A. Bendersky, An Isotropic Glass Phase in Al-Fe-Si Formed by a First Order Transition, Mat. Res. Soc. Symp. Proc., 2004, 806, p MM2.7.1-MM2.7.5

5. J. Hafner, Ab Initio Density-Functional Calculations In Materials Science: From Quasicrystals Over Microporous Catalysts to Spintronics, J. Phys. Condense. Mater., 2010, 22, p 384205

6. B. Grushko and T.Ya Velikanova, Stable and Metastable Quasicrystals in Al-Based Alloy Systems with Transition Metals, J. Alloys Comp., 2004, 367, p 58

7. G. Shao, V. Varsani, and Z. Fan, Thermodynamic Modelling of the Y-Zn and Mg-Zn-Y Systems, Calphad, 2006, 30, p 286-295

8. www.ctcms.nist.gov/potentials/. Last accessed 2 Feb 2012 